\title{
SPACE OBJECT TRAFFIC CONTROL
}

\section{STEPHEN H. KNOWLES}

Naval Space Surveillance Center, Dahlgren, VA U.S.A.

\begin{abstract}
The task of avoiding collisions between orbiting objects requires more than a statistical description of the objects' positions. The paper describes the general procedures used for such cataloging, together with an indication of the accuracy required for orbital collision avoidance.
\end{abstract}

